\title{
Quiz intensiv Stellen Sie die Diagnose!
}

Fallbeschreibung Eine 43-jährige Patientin stellt sich initial mit therapieresistenten Schmerzen im distalen rechten Oberschenkel beim Orthopäden vor. Nach durchgeführter Bildgebung besteht der hochgradige Verdacht auf Knochenmetastasen und pulmonale Infiltration. Zur Lokalisation des Primarius und weiteren Tumorabklärung erfolgt die stationäre Aufnahme.

Im Rahmen dieser Abklärung ist eine endobronchiale UltraschallUntersuchung mit Biopsie der mediastinalen Lymphknotenpakete geplant. Bei dieser Untersuchung in totaler i. v. Anästhesie (TIVA) und Jet-Ventilation erleidet die Patientin einen schweren Asthmaanfall (Abfall der $\mathrm{O}_{2}$-Sättigung bis auf $<40 \%$ ). Die Untersuchung wird sofort abgebrochen, die Patientin mit

- 250 mg Prednisolon,

- insgesamt 0,36 mg Reproterol,

- fraktioniert $600 \mathrm{mg}$ Ketamin und

- Epinephrin per inhalationem

behandelt.

Die Narkose wird mit Sevofluran fortgesetzt und vertieft. Die Oxygenierung kann nur unter hohen Beatmungsdrücken (inspiratorischer Beatmungsdruck [ $\mathrm{p}_{\text {insp }}$ ] zeitweise bis $45 \mathrm{mbar}$ ) stabilisiert werden (arterielle Blutgasanalyse: $\mathrm{pO}_{2} 65 \mathrm{mmHg} ; \mathrm{pCO}_{2}$ $73 \mathrm{mmHg} ; \mathrm{pH} 7,17 ; \mathrm{SO}_{2} 85 \%$ unter $\mathrm{FiO}_{2}$ 1,0). Es erfolgt der notfallmäßige Transport auf die Intensivstation und nach Anlage eines ZVK die Anfertigung eines Röntgen-Thorax ( $\bullet$ Abb. 1).

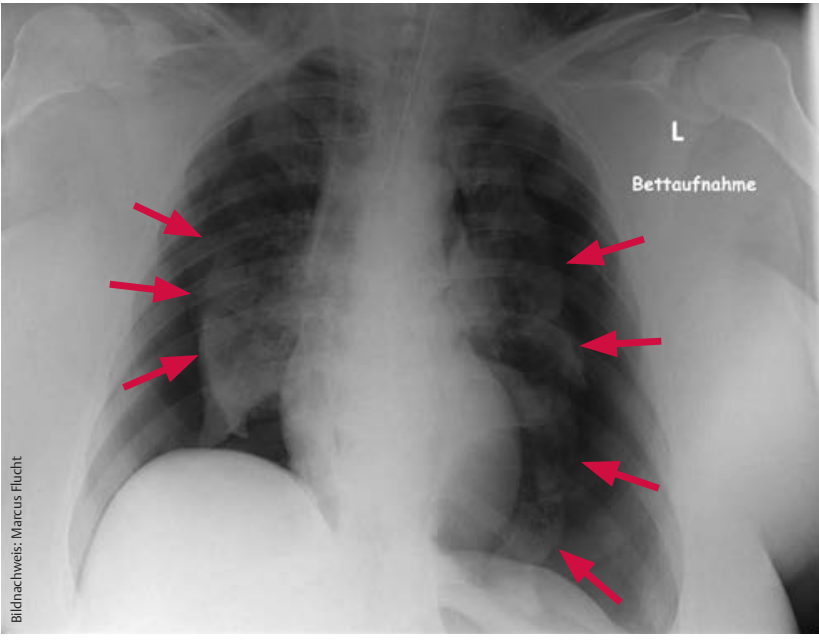

Abb. 1 Röntgen-Thorax anterior-posterior bei Aufnahme auf die Intensivstation.

- Wie lautet Ihre Diagnose?

- Welche Differenzialdiagnosen müssen in Betracht gezogen werden?

- Welche Therapie ist indiziert?

Die Auflösung finden Sie auf der nächsten Seite 


\section{Quiz intensiv Stellen Sie die Diagnose!}

\section{Auflösung}

\section{Diagnose}

- Barotrauma der Lunge durch Überdruckbeatmung (Jet-Ventilation) mit beidseitigem Pneumothorax.

\section{Differenzialdiagnose \\ - Hypoventilation \\ - Pneumothorax \\ - Pneumomediastinum \\ - subkutanes Emphysem \\ - Pneumoperitoneum \\ - Magendistension}

\section{Korrespondenzadresse}

Marcus Flucht

Abteilung für Anästhesie

St. Elisabeth-Krankenhaus jülich

Kurfürstenstr. 22, 52428 Jülich

Prof. Dr. med. Tobias Schürholz

Klinik für Operative Intensivmedizin und Intermediate Care

Universitätsklinikum der RWTH Aachen

Pauwelsstr. 30, 52074 Aachen

E-Mail: tschuerholz@ukaachen.de

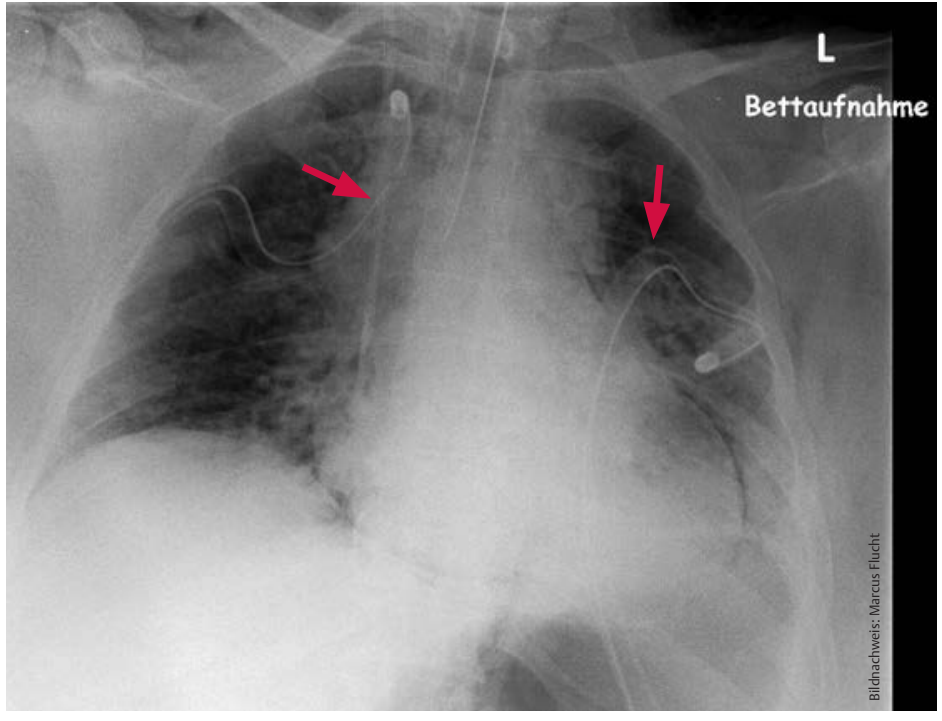

Abb. 2 Röntgen-Thorax anterior posterior nach Anlage der Thoraxdrainagen (durch Pfeile markiert).

Therapie Nach der radiologischen Diagnose „Pneumothorax beidseits“ erhält die Patientin rechts und links eine Thoraxdrainage in Monaldiposition. Hierunter bessert sich der Zustand schlagartig, sodass sie noch am selben Tag problemlos extubiert werden kann (॰ Abb. 2).

Fazit Bei der Jet-Ventilation werden niedrige Atemhubvolumina mit hoher Frequenz und hohem Druck in die Trachea appliziert. Damit unterscheidet sie sich von konventioneller Beatmung und findet v. a. bei endoskopischen Untersuchungen und Eingriffen an den Bronchien Anwendung.

Als an die Bedürfnisse des Patienten anzupassende Parameter sind v. a. die $\mathrm{O}_{2}$-Konzentration und der Arbeitsdruck entscheidend [1]. Der Großteil der Untersuchungen verläuft dabei ohne Komplikationen [1]. Die häufigsten Komplikationen sind jedoch kardiale Arrhythmien [2]. Mögliche weitere Komplikationen wie z.B. Hypoventilation, Pneumothorax, Pneumomediastinum, subkutanes Emphysem, Pneumoperitoneum und Magendistension sind bei klinischer Verschlechterung zu bedenken.

\section{Literaturverzeichnis}

1 Kern M, Niemeyer D, Kerner T et al. Anästhesie in der interventionellen Pneumologie - Bronchoskopie und Jet-Ventilation. Anasthesiol Intensivmed Notfallmed Schmerzther 2015; 50: 8-18

2 Kern M, Niemeyer D, Kerner T et al. Anästhesie in der interventionellen Pneumologie - Endoskopische Interventionen: Teil 2. Anasthesiol Intensivmed Notfallmed Schmerzther 2015; 50: 308 314

Beitrag online zu finden unter http://dx. doi.org/10.1055/s-0042-101778 